\title{
Effect of physiological heart rate changes on left ventricular dimensions and mitral blood flow velocities in the normal fetus
}

\author{
P.B. Tsyvian ${ }^{\text {a }}$, K.V. Malkin ${ }^{\mathrm{a}}$, J.W. Wladimiroff* ${ }^{\mathrm{b}}$ \\ a Biophysical Laboratory, Mother and Child Care Institute, Yekaterinburg, Russian Federation \\ ${ }^{b}$ Department of Obstetrics and Gynecology, Academic Hospital Rotterdam-Dijkzigt, Eramus University, \\ Dr. Molewaterplein 40, 3015 GD Rotterdam. The Netherlands
}

Received 13 June 1994; revision received 3 September 1994; accepted 4 October 1994

\begin{abstract}
M-mode echo recordings of the left ventricle (LV) and inflow LV Doppler velocimetry were performed in nine normal fetuses at a gestational age of 36-39 weeks. In each fetus $\sim 80$ consecutive cardiac cycles were digitized. The duration of each cardiac cycle $(T)$ and the corresponding end-diastolic (EDD), end-systolic (ESD) dimensions of LV or the peak velocity of early (E) and late atrial (A) mitral flow parameters was calculated. The role of sonographic parameters on current $(\mathrm{Tn})$ and preceding $(\mathrm{Tn}-1)$ cardiac cycles was assessed using linear regression. Significant dependency of ventricular EDD and transmitral A peak velocity upon Tn was demonstrated. We speculate that atrial systole has an important role to play in the beat-to-beat regulation of fetal stroke volume.
\end{abstract}

Keywords: Ventricular dimensions; Atrial systole; Heart rate

\section{Introduction}

Evaluation of cardiac function generally concentrates on three major controlling mechanisms. These are the diastolic muscle fibre length (the Frank-Starling mechanism), neural and other influences that affect force independently of muscle length (inotropic mechanisms) and the load that the ventricle must overcome in ejecting blood (afterload). The relationship between contraction frequency and force is also an important component of cardiac function description.

\footnotetext{
* Corresponding author, Tel: 010463 3632; Fax: 0104635826.
} 
If an isolated strip of cardiac muscle is fixed at its two ends and electrically stimulated at different rates the developed force may be assessed according to the so-called force-frequency relation [1]. Force-frequency relation is determined by the mechanisms of excitation-contraction coupling of myocardial cells. These mechanisms provide the transmission and conversion of electrical signal parameters (action potential) into sufficient intracellular $\mathrm{Ca}++$ ion concentration changes [2].

The force of cardiac contraction can be directly measured in isolated muscle. In the intact heart the problem is more complicated because the equivalent measurement cannot be made directly. The relationship between stroke volume and heart rate in the intact heart is a more complex phenomenon than the force-frequency relation in the isolated muscle, since stroke volume is not only dependent upon myocardial contractility, but also upon preload and afterload.

Recent studies in man have shown that rate dependency of aortic pulse pressures in adults with atrial fibrillation was mainly determined by chronoinotropic regulation or force-frequency relationship [3]. Experimental animal studies have demonstrated that force-frequency phenomena are poorly developed in the fetal myocardium and fetal heart function according to the Frank-Starling mechanism $[4,5]$. Therefore, diastolic filling may be the main determinant of fetal cardiac performance during heart rate changes.

The aim of the present study was to evaluate the influence of heart rate on cardiac performance and mitral inflow patterns during physiological heart rate changes in normal fetuses.

\section{Materials and methods}

A total of 32 examinations were carried out in nine fetuses between 36 and 39 weeks of gestation. This particular gestational age period was chosen to ensure fetal maturity. All fetuses displayed normal growth, normal umbilical Doppler flow velocity waveforms and no congenital anomalies. Real-time ultrasound scans were made with a $3.5-\mathrm{MHz}$ linear-array transducer and were combined with $\mathrm{M}$-mode recording (Aloka SSD 650). After identification of normal cardiac anatomy an acceptable four chamber view of the heart was obtained. The M-mode image was obtained at right angles to the left and right ventricle at the level of the mitral and tricuspid valve leaflets and was recorded on video tape and after examination documented on a strip chart. From well-defined $\mathrm{M}$-mode traces with no disturbances of rhythm and showing neither fetal respiration nor body movements, $\sim 80$ consecutive cardiac cycles were digitized. Pulsed Doppler flow velocity tracings were obtained at the level of the mitral valve immediately after the M-mode recording. The interrogation angle was always kept below $10^{\circ}$. Eighty consecutive cardiac cycles were digitized under the same conditions. Analysis of $\mathrm{M}$-mode recordings and Doppler waveforms was performed from hardcopies using a personal computer, Apricot XEN-S, linked to a graphic tablet. In each cardiac cycle the endocardial borderlines (contours) of the left ventricle (M-mode) and $\mathrm{E}$ - and A-waves (Doppler) were traced manually. Each cardiac cycle was stored in a computer memory for data analysis.

Measurements included duration of each cardiac cycle ( $T$, s), end-diastolic (EDD, 
$\mathrm{cm}$ ) and end-systolic (ESD, $\mathrm{cm}$ ) left ventricular dimensions, fractional shortening $(\% \mathrm{FS})$, transmitral peak velocity during early diastole $(\mathrm{E}, \mathrm{cm} / \mathrm{s})$ and transmitral peak velocity during atrial contraction $(\mathrm{A}, \mathrm{cm} / \mathrm{s})$. A series of $N$ consecutive cardiac cycles (T) and a corresponding series of consecutive left ventricular echocardiographic parameters (P) were entered into the personal computer. The computer programme automatically calculates mean values for each series, dispersion and coefficients of variation. The level of statistical significance chosen was $P<0.05$.

Since no fetal body or breathing movements occurred during the period of registration, the venous return and afterload parameters were considered constant. Simultaneous recording of M-mode/transmitral Doppler waveforms and aortic outflow/inferior vena cava collapse index was not feasible, however, Doppler tracings were obtained immediately after the $\mathbf{M}$-mode registrations. It was thus assumed that fetal hemodynamic conditions during these recordings were similar. To obtain the maximal heartrate variability, recordings were chosen with a coefficient of variation of cardiac interval duration $\geq 10 \%$.

The dependence of current echocardiographic parameters upon the duration of cardiac intervals and previous echocardiographic parameters was described in general form by the following regression equation:

$$
\mathrm{Pn}=a 1 \mathrm{Tn}+a 2 \mathrm{Tn}-1+a 3 \mathrm{Pn}-1
$$

where $\mathrm{Pn}$ and $\mathrm{Pn}-1$ represent the current and previous dimension of the Doppler and M-mode parameters and Tn and Tn - 1 represent the current and previous cardiac interval. $a 1$ is the coefficient of linear regression for relationship Pn-Tn known in physiology as 'mechanical restitution', $a 2$ is the linear regression coefficient for 'postextrasystolic potentiation' or Pn- $\mathrm{Tn}-1$ relation and $a 3$ is the auto-regression coefficient between current and preceding dimension parameters and represents the influence of preceding cardiac contraction upon the current contraction or 'memory of the system'.

\section{Results}

Table 1 shows the findings obtained from the left ventricle of the investigated group of fetuses. At a mean heart rate of $142 \pm 12$ (1 S.D.) bpm, left ventricular enddiastolic and end-systolic dimensions were $1.46 \pm 0.14$ and $1.02 \pm 0.08 \mathrm{~cm}$, respec-

Table 1

Echocardiographic data of normal fetuses (mean \pm S.D.)

Heart rate (bpm)

Left ventricular EDD $(\mathrm{cm})$

Left ventricular ESD $(\mathrm{cm})$

LV fractional shortening (\%)

Transmitral E-peak velocity $(\mathrm{cm} / \mathrm{s})$

Transmitral A-peak velocity $(\mathrm{cm} / \mathrm{s})$

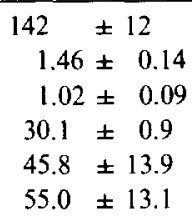


Table 2

Mean values for regression coefficients $(n=32)$

\begin{tabular}{|c|c|c|c|}
\hline Parameters as $\mathrm{Pn}$ & $a \mathbf{l}$ & $a 2$ & $a 3$ \\
\hline LV fractional shortening & $0.51 \pm 0.03^{* *}$ & $-0.33 \pm 0.01^{*}$ & $0.12 \pm 0.03$ \\
\hline Left ventricular EDD & $0.46 \pm 0.03^{* *}$ & $-0.27 \pm 0.01^{*}$ & $0.10 \pm 0.02$ \\
\hline Left ventricular ESD & $0.11 \pm 0.01$ & $-0.09 \pm 0.01$ & $0.72 \pm 0.04^{* * *}$ \\
\hline Transmitral E peak velocity & $0.17 \pm 0.02$ & $-0.07 \pm 0.03$ & $-0.09 \pm 0.03$ \\
\hline Transmitral A peak velocity & $-0.52 \pm 0.04^{* *}$ & $-0.29 \pm 0.05$ & $0.50 \pm 0.02^{* *}$ \\
\hline
\end{tabular}

" $P<0.05 ;{ }^{* *} P<0.01 ;{ }^{* * *} P<0.001$

$a$, coefficient of linear regression for relationship Pn-Tn (current sonographic parameter current cardiac interval); $a 2$, coefficient of linear regression for relationship $\mathrm{Pn}-\mathrm{Tn}-1$ (current sonographic parameter preceding cardiac interval); $a 3$, autoregression coefficient between current $(\mathrm{Pn})$ and preceding $(\mathrm{Pn}-1)$ sonographic parameters.

tively. The mean values for transmitral $\mathrm{E}$ and $\mathrm{A}$ peak velocities were $45.8 \pm 13.9$ and $55.0 \pm 13.1 \mathrm{~cm} / \mathrm{s}$, respectively.

The influence of current and preceding cardiac interval duration, and preceding echocardiographic parameters on current echocardiographic parameters are represented in coefficient of regression equations. The coefficients (mean \pm S.D.) for dimension and Doppler parameters are shown in Table 2. A significant dependency of end-diastolic dimensions and fractional shortening upon current and preceding cardiac intervals was observed. Coefficients $a 1$ for fractional shortening and enddiastolic dimension were significant: $0.51 \pm 0.03$ and $0.46 \pm 0.03(P<0.01)$, respectively. Both fractional shortening and end-diastolic diameter were inversely correlated to $\mathrm{Tn}-1$ or preceding cardiac interval. The mean values for $a 2$ coefficients of linear regression were $-0.33 \pm 0.01 \quad(P=0.04)$ and $-0.27 \pm 0.01$ $(P=0.03)$. Coefficient $a 3$ was significant only for the end-systolic dimension of the left ventricle $(0.72 \pm 0.04, P<0.001)$. There was no cross-correlation between ventricular end-systolic dimension and duration of current and preceding cardiac intervals. There was also no cross-correlation between transmitral E peak velocity and cardiac cycle length, but the amplitude of transmitral A peak velocity displayed a negative correlation $(-0.52 \pm 0.04 ; P<0.01)$ with cardiac cycle length.

\section{Discussion}

Our results suggest a significant influence of current and preceding cardiac intervals upon ventricular end-diastolic dimension and fractional shortening, i.e. the amplitude of stroke volume is determined by the end-diastolic volume and by the duration of cardiac intervals. Therefore, our results show that in normal fetuses the 'stroke volume - rate' relationship of the heart functions according to the FrankStarling law.

The inverse correlation between fractional shortening and end-diastolic dimension and $\mathrm{Tn}-1$ or preceding cardiac interval corresponds to a well-known negative cor- 
relation between postextrasystolic contraction and the duration of the extrasystolic cardiac interval in the mammalian heart $[1,4]$.

It is known that changes of ventricular end-diastolic volume in case of constant afterload and myocardial contractility, lead to variation in stroke volume without significant changes in end-systolic volume [6]. The lack of correlation between endsystolic dimension and current and preceding cardiac intervals and the significant positive auto-correlation of end-systolic dimension demonstrate the high stability of this dimension and therefore the absence of significant changes in afterload and level of myocardial contractility during the episodes of heart rate variations. This suggests that chronoinotropic phenomena are not fully developed in the fetal ventricular myocardium. The lack of any correlation between transmitral E-peak and cardiac interval duration as well as the presence of a significant negative correlation between Doppler transmitral A-peak velocity and current cardiac interval shows that atrial systole plays an important role in the rhythm dependent regulation of cardiac output.

Several explanations can be put forward for the interaction between heart rate variations and atrial contribution in left ventricular filling. Firstly, an increase in heart rate might lead to impaired left ventricular relaxation and an increase in the contribution of left atrial systole in ventricular filling. However, our data failed to show a decrease in transmitral E-peak velocity. Moreover, it appears unlikely that physiological variations in heart rate can induce a significant change in ventricuiar relaxation. Secondly, the shortening of the diastolic filling time as heart rate increases, may result in incomplete left atrial emptying before the onset of atrial contraction. Therefore, a greater left atrial volume may enhance atrial performance in Frank-Starling mechanism fashion. Thirdly, the force-frequency relation and other chronoinotropic phenomena could apply to the left atrium resulting in a greater beat-to-beat variation in atrial contractile force than in ventricular contractile force. It is possible that either of the latter two mechanisms may be valid, and as they are not mutually exclusive may function in cooperation.

Although in this investigation we tried to record echocardiographic parameters under relatively constant fetal hemodynamic conditions (absence of breathing and body movements) the linear representation of the relationship between $\mathrm{Pn}, \mathrm{Tn}$, $\mathrm{Tn}-1$ and $\mathrm{Pn}-1$ may be considered as a significant simplification. The obtained correlations, however, demonstrate that atrial systole plays an important role in beat-to-beat regulation of left ventricular stroke volume of normally growing fetuses.

\section{Acknowledgement}

This study was supported by the Dutch Research Council, NWD no. 0713091.

\section{References}

[1] Koch-Weser, J. and Blinks, J. (1963): The influence of the interval between beats on myocardial contractility. Pharmacol. Rev., 15, 601-652. 
[2] Morad, M. and Goldman, Y. (1973): Excitation-contraction coupling in heart muscle: membrane control of development of tension. Prog. Biophys. Mol. Biol., 27, 257-313.

[3] Pfeiffer, K.P., Kenner, T. and Schaefer, J. (1984): Application of statistical methods for the analysis of interval related cardiac performance variations during cardiac arrhythmia in man. Cardiovasc. Res., 18, 80-98.

[4] Maylie, J. (1982): Excitation-contraction coupling in neonatal and adult myocardium of the cat. Am. J. Physiol., 242, 834-843.

[5] Kirkpatrick, S.E., Pitlick, P.T., Naliboff, J. and Freidman, W.F. (1976): Frank-Staring relationship as an important determinant of fetal cardiac output. Am. J. Physiol., 231, 495-500.

[6] Suga, H., Kitabatake, A. and Sagawa, K. (1979): End-systolic pressure determines stroke volume from fixed end-diastolic volume in the isolated canine left ventricle under a constant contractile state. Circ. Res., 44, 238-249. 